\title{
Highlights from the ARGO-YBJ experiment
}

\author{
G. Aielli ${ }^{\text {a,b }}$, C. Bacci ${ }^{\text {c,d }}$, B. Bartoli ${ }^{\text {e,f }}$, P. Bernardini ${ }^{\text {g,h }}$, X.J. Bi ${ }^{i}$, C. Bleve ${ }^{\text {g,h }}$, P. Branchini ${ }^{\text {d }}$, A. Budano ${ }^{\text {d }}$,
} S. Bussino ${ }^{\text {c,d }}$, A.K. Calabrese Melcarne ${ }^{\text {j, P. Camarri }}{ }^{\text {a,b,* }}$, Z. Cao $^{\mathrm{i}}$, A. Cappa ${ }^{\mathrm{k}, \mathrm{l}}$, R. Cardarelli ${ }^{\mathrm{b}}$, S. Catalanotti ${ }^{\text {e,f }}$, C. Cattaneo $^{\mathrm{m}}$, P. Celio ${ }^{\text {c,d }}$, S.Z. Chen ${ }^{\mathrm{i}}$, T.L. Chen ${ }^{\mathrm{s}}$, Y. Chen ${ }^{\mathrm{i}}$, N. Cheng ${ }^{\mathrm{i}}$, P. Creti ${ }^{\text {h }}$, S.W. Cui ${ }^{\text {, }}$, B.Z. Dai ${ }^{\mathrm{p}}$,

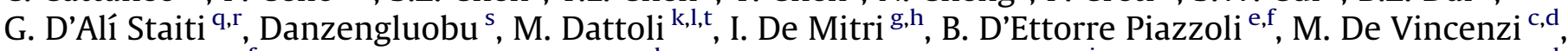

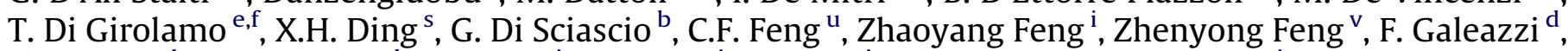
P. Galeotti ${ }^{\text {k,t }}$, R. Gargana ${ }^{\mathrm{d}}$, Q.B. Gou ${ }^{\mathrm{i}}$, Y.Q. Guo ${ }^{\mathrm{i}}$, H.H. He ${ }^{\mathrm{i}}$, Haibing Hu ${ }^{\mathrm{s}}$, Hongbo Hu ${ }^{\mathrm{i}}$, Q. Huang ${ }^{\mathrm{v}}$,

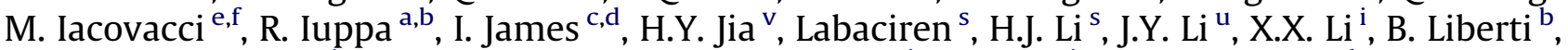

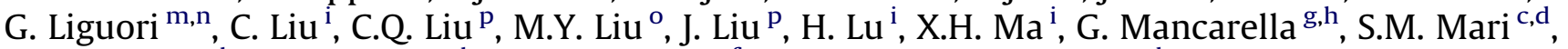
G. Marsella w,h $^{\text {, D. Martello }}{ }^{\text {g,h }}$, S. Mastroianni ${ }^{\text {f }}$, X.R. Meng ${ }^{\text {s }}$, P. Montini ${ }^{\text {c,d }}$, C.C. Ning ${ }^{\text {s }}$, A. Pagliaro ${ }^{\text {x,r }}$, M. Panareo ${ }^{\text {w,h }}$, L. Perrone ${ }^{\text {w,h }}$, P. Pistilli ${ }^{\text {c,d }}$, X.B. Qu ${ }^{\text {u }}$, E. Rossi ${ }^{\text {f }}$, F. Ruggieri ${ }^{\text {d }}$, L. Saggese ${ }^{\text {e,f }}$, P. Salvini ${ }^{\mathrm{m}}$, R. Santonico ${ }^{\text {a,b }}$, P.R. Shen ${ }^{i}$, X.D. Sheng ${ }^{i}$, F. Shi ${ }^{\text {i }}$, C. Stanescu ${ }^{\text {d }}$, A. Surdo ${ }^{\text {h }, ~ Y . H . ~ T a n ~}{ }^{\text {i }}$, P. Vallania ${ }^{\text {k,l }}$,

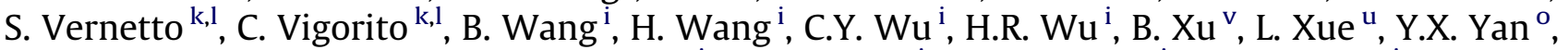
Q.Y. Yang ${ }^{\mathrm{p}}$, X.C. Yang ${ }^{\mathrm{p}}$, A.F. Yuan ${ }^{\mathrm{s}}$, M. Zha ${ }^{\mathrm{i}}$, H.M. Zhang ${ }^{\mathrm{i}}$, JiLong Zhang ${ }^{\mathrm{i}}$, JianLi Zhang ${ }^{\mathrm{i}}$, L. Zhang ${ }^{\mathrm{p}}$,

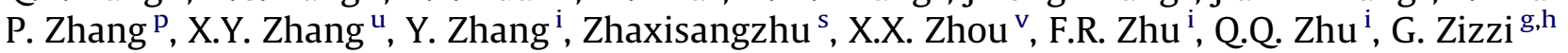

a Dipartimento di Fisica, dell'Università “Tor Vergata”, via della Ricerca Scientifica 1, 00133 Roma, Italy

b Istituto Nazionale di Fisica Nucleare, Sezione di Roma Tor Vergata, via della Ricerca Scientifica 1, 00133 Roma, Italy

'Dipartimento di Fisica, dell'Università “Roma Tre", via della Vasca Navale 84, 00146 Roma, Italy

d Istituto Nazionale di Fisica Nucleare, Sezione di Roma 3, via della Vasca Navale 84, 00146 Roma, Italy

e Dipartimento di Fisica, dell'Università di Napoli, Complesso Universitario di Monte Sant'Angelo, via Cintia, 80126 Napoli, Italy

${ }^{\mathrm{f}}$ Istituto Nazionale di Fisica Nucleare, Sezione di Napoli, Complesso Universitario di Monte Sant'Angelo, via Cintia, 80126 Napoli, Italy

${ }^{g}$ Dipartimento di Fisica, dell'Università del Salento, via per Arnesano, 73100 Lecce, Italy

${ }^{\mathrm{h}}$ Istituto Nazionale di Fisica Nucleare, Sezione di Lecce, via per Arnesano, 73100 Lecce, Italy

${ }^{\mathrm{i}}$ Key Laboratory of Particle Astrophyics, Institute of High Energy Physics, Chinese Academy of Science, P.O. Box 918, 100049 Beijing, PR China

${ }^{\mathrm{j}}$ Istituto Nazionale di Fisica Nucleare, CNAF, viale Berti-Pichat 6/2, 40127 Bologna, Italy

${ }^{\mathrm{k}}$ Istituto Nazionale di Fisica Nucleare, Sezione di Torino, via P. Giuria 1, 10125 Torino, Italy

${ }^{1}$ Istituto di Fisica dello Spazio Interplanetario dell'Istituto Nazionale di Astrofisica, corso Fiume 4, 10133 Torino, Italy

${ }^{\mathrm{m}}$ Istituto Nazionale di Fisica Nucleare, Sezione di Pavia, via Bassi 6, 27100 Pavia, Italy

n Dipartimento di Fisica Nucleare e Teorica, dell'Università di Pavia, via Bassi 6, 27100 Pavia, Italy

${ }^{\circ}$ Hebei Normal University, Shijiazhuang 050016, Hebei, China

p Yunnan University, 2 North Cuihu Rd, 650091 Kunming, Yunnan, PR China

${ }^{\mathrm{q}}$ Università degli Studi di Palermo, Dipartimento di Fisica e Tecnologie Relative, Viale delle Scienze, Edificio 18, 90128 Palermo, Italy

${ }^{\mathrm{r}}$ Istituto Nazionale di Fisica Nucleare, Sezione di Catania, Viale A. Doria 6, 95125 Catania, Italy

s Tibet University, 850000 Lhasa, Xizang, PR China

t Dipartimento di Fisica Generale, dell'Università di Torino, via P. Giuria 1, 10125 Torino, Italy

u Shandong University, 250100 Jinan, Shandong, PR China

v South West Jiaotong University, 610031 Chengdu, Sichuan, PR China

${ }^{\mathrm{w}}$ Dipartimento di Ingegneria dell'Innovazione, Università del Salento, 73100 Lecce, Italy

${ }^{\mathrm{x}}$ Istituto di Astrofisica Spaziale e Fisica Cosmica di Palermo, Istituto Nazionale di Astrofisica, via Ugo La Malfa 153, 90146 Palermo, Italy

\section{A R T I C L E I N F O}

Available online 3 December 2010

Keywords:

Resistive Plate Chambers

Extensive air showers

\section{A B S T R A C T}

The ARGO-YBJ experiment at YangBajing in Tibet (4300 $\mathrm{m}$ a.s.l.) has been taking data with its full layout since October 2007. Here we present a few significant results obtained in gamma-ray astronomy and cosmic-ray physics. Emphasis is placed on the analysis of gamma-ray emission from point-like sources (Crab Nebula, MRK 421), on the preliminary limit on the antiproton/proton flux ratio, on the large-scale

\footnotetext{
* Corresponding author at: Università degli Studi di Roma "Tor Vergata", Dipartimento di Fisica, via della Ricerca Scientifica 1, 00133 Roma, Italy. Tel.: +39 0672594302 ; fax: +3906 2023507

E-mail address: paolo.camarri@roma2.infn.it (P. Camarri).
} 
Ground-based $\gamma$-ray astronomy Cosmic-ray physics cosmic-ray anisotropy and on the proton-air cross-section. The performance of the detector is also discussed, and the perspectives of the experiment are outlined.

(c) 2010 Elsevier B.V. All rights reserved.

\section{Introduction}

The requirement of lowering the detection energy threshold of primaries (and therefore to increase the detector sensitivity) in a ground-based experiment can be fulfilled by a full-coverage detector at high altitude. Resistive Plate Chambers (RPCs) [1] were chosen for this purpose due to their high time resolution (between 1 and 2 ns, definitely suitable for a precise reconstruction of an extensive air shower (EAS) front) and to their reasonable cost which makes them a perfect choice for a large-area detector. The ARGO-YBJ experiment [2] exploits the features of RPCs for the detection of extensive air showers. ARGO-YBJ was designed to investigate a large number of topics in astrophysics and cosmic-ray physics:

- $\gamma$-ray astronomy (search for point-like sources above few hundreds of $\mathrm{GeV}$ );

- search for very high-energy (VHE) tails of $\gamma$-ray bursts above $\sim 1 \mathrm{GeV}$;

- cosmic-ray physics;

- Sun and heliosphere physics.

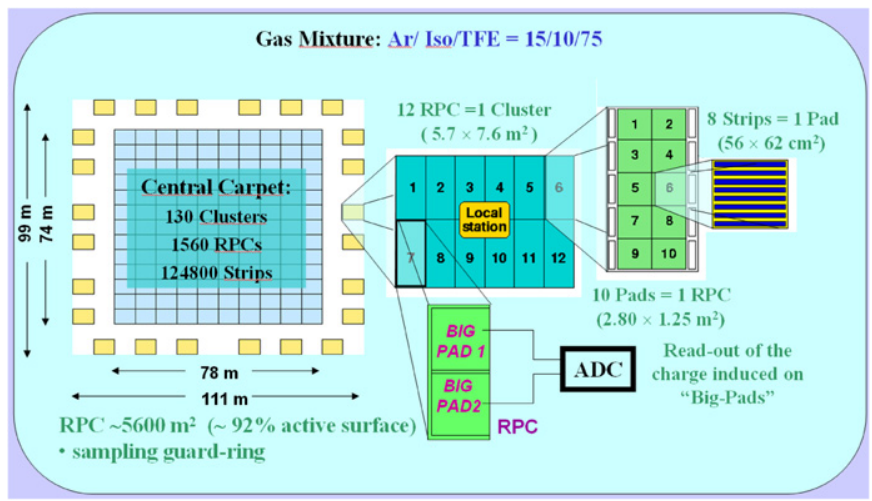

Fig. 1. Schematic drawing of the ARGO-YBJ experimental setup, with the details of the structure of one cluster and one pad.

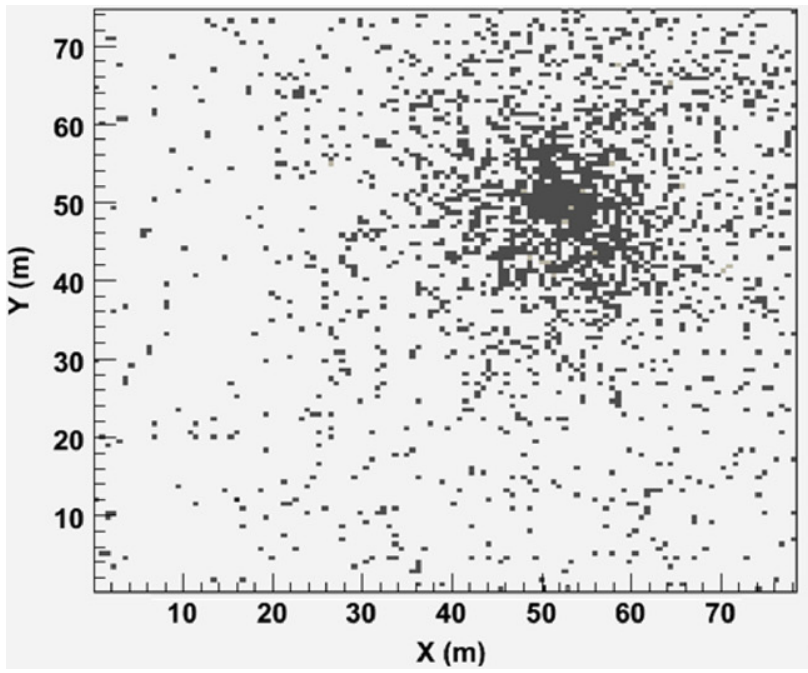

\section{The ARGO-YBJ detector}

The ARGO-YBJ experiment is installed at Yangbajing (PR of China), $4300 \mathrm{~m}$ a.s.l., longitude $90^{\circ} 31^{\prime} 50^{\prime \prime}$ East, latitude $30^{\circ} 06^{\prime} 38^{\prime}$ North. The detector [3] is composed of 1560 RPCs $\left(2.80 \times 1.25 \mathrm{~m}^{2}\right.$ each) in the central full-coverage region, plus 276 RPCs in the "guard ring" area close to the outer wall of the experimental building, as shown in Fig. 1.

Each of the ARGO-YBJ RPCs is composed of two 2-mm thick plates of plastic laminate with bulk resistivity of about $10^{12} \Omega \mathrm{cm}$, enclosing a 2 -mm thick gas gap. A grid of PET spacers is placed between the plates to fix the gap width over the whole chamber area. The electrical contacts for the high voltage are provided by thin graphite layers on the outer sides of the plates, protected by a PET foil. The readout strip panel is placed on the ground face of the RPC. Eighty copper strips $(6.5 \times$ $62 \mathrm{~cm}^{2}$ each) collect and transmit the signals generated by the passage of charged particles across the RPC gas gap. At the outer end of the strips, the front-end electronic boards [4] suitably amplify and shape the signals for subsequent processing. A "pad" is the basic acquisition unit of the ARGO-YBJ detector, and it provides the logical OR of the eight strips. Fig. 2 shows a reconstructed event, with the space pattern of the hits (left) and the space-time structure of the shower front.

On the other side of the gas volume, two copper "big pads" are placed in order to collect the analog signal from the detector. The analog readout of the ARGO-YBJ detector was put into operation in 2009. This information will be extremely useful for studying events generated by primaries with energy $\gtrsim 100 \mathrm{TeV}$, when the digital information from the readout strip starts to become saturated and it would be hard to reconstruct the position of the air-shower core on the carpet.

Fig. 3 shows a simulated event with primary energy of $10^{15} \mathrm{eV}$ : the digital information (left) cannot easily identify the core position, while the analog information from the big pads (right) clearly does. This technique will allow to extend the ARGO-YBJ energy range above $1 \mathrm{PeV}$.

ARGO-YBJ started taking data with its complete layout in October 2007. Since then it went on almost uninterruptedly with a duty cycle of $90 \%$ and trigger rate of $3.6 \mathrm{kHz}$.

Fig. 2. ARGO-YBJ reconstructed event. Left: space map of the hit pads on the central carpet. Right: space-time structure of the shower front. 

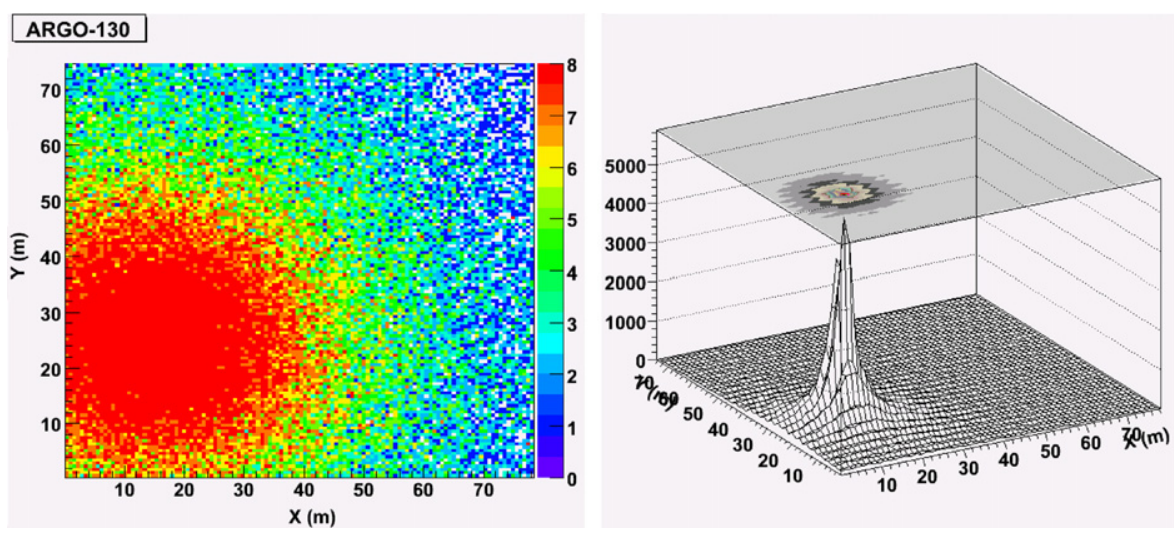

Fig. 3. Simulated ARGO-YBJ event for a proton with primary energy of 1 PeV. Left: space map of the hit pads on the detector. Right: bi-dimensional space profile for the response of the analog readout.

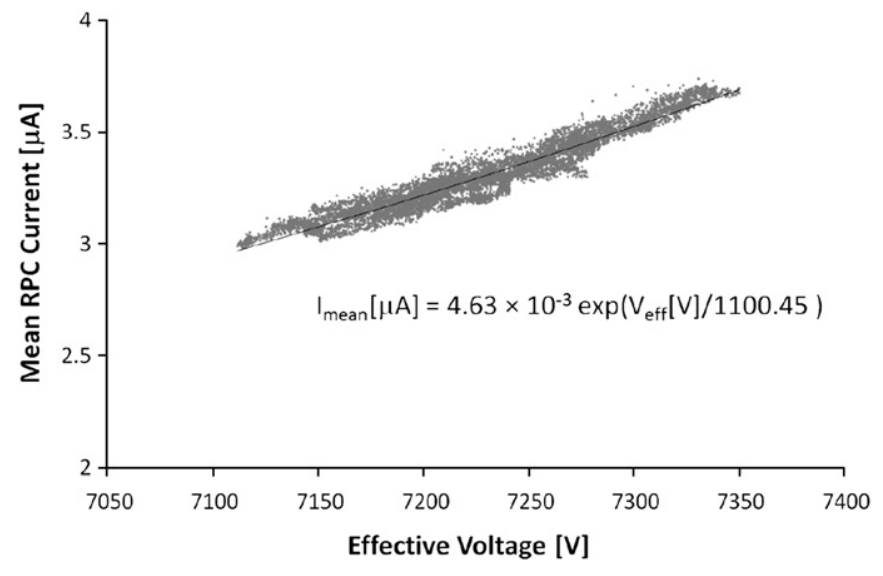

Fig. 4. Correlation plot of the average RPC chamber current versus the effective voltage on the detectors. The best fit with an exponential function is superimposed to the plot.

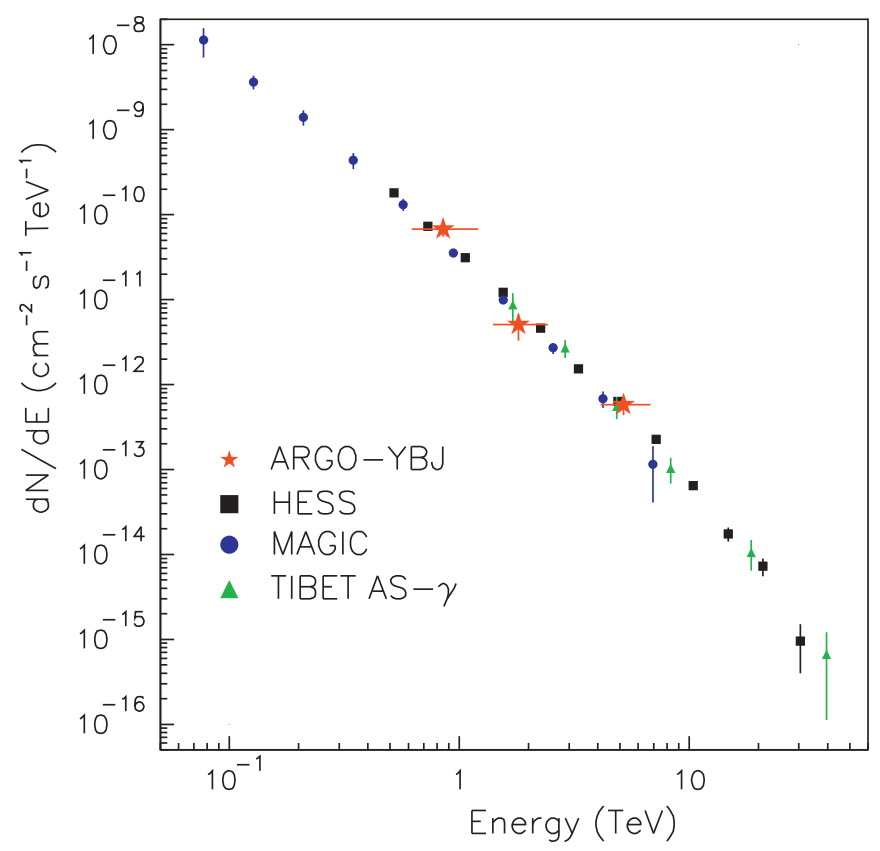

Fig. 5. Experimental energy spectrum for the $\gamma$-rays from the Crab Nebula in the range from a few $\mathrm{GeV}$ up to $50 \mathrm{TeV}$. The ARGO-YBJ experimental points are in perfect agreement with the known results from HESS, MAGIC and Tibet AS- $\gamma$.

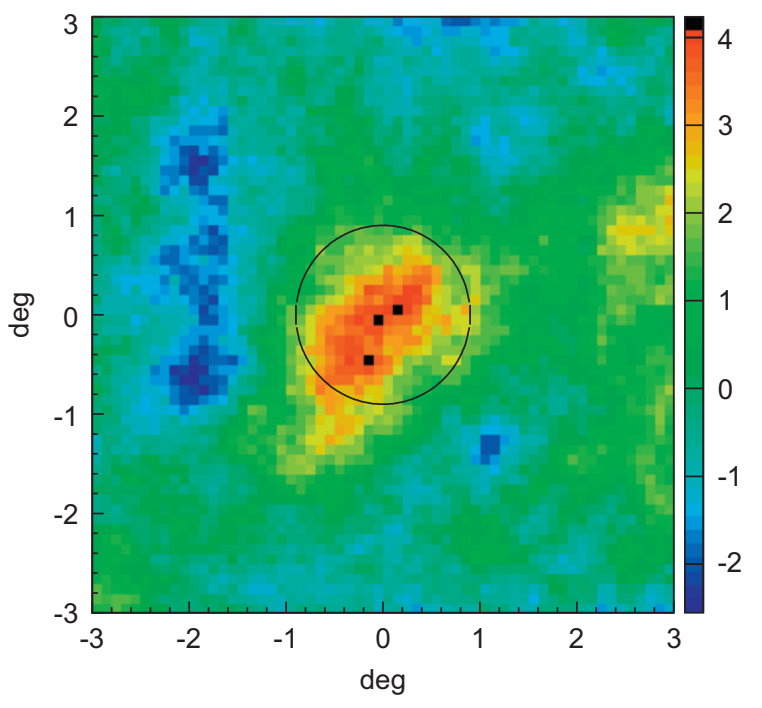

Fig. 6. ARGO-YBJ significance map of MRK 421 during the flare of June 11-13, 2008. The maximum significance was 4.2 standard deviations.

The environmental and operational parameters of the ARGO-YBJ detector are constantly monitored. In Fig. 4 the correlation plot of the mean chamber current versus the effective voltage [5] acting on the ARGO-YBJ RPCs at the same time is shown. The correlation is maximized if a delayed effect of the temperature changes on the effective voltage is accounted for [6]. The resulting high degree of correlation confirms the stability of the whole detector.

\section{ARGO-YBJ results in gamma-ray astronomy}

The analysis of the ARGO-YBJ data in $\gamma$-ray astronomy in the first two years of running was mainly focused on the study of known sources, on the study of flares from active galactic nuclei (AGN), and on the limits on very high-energy flux from $\gamma$-ray bursts. The background was evaluated by using two independent, widely established procedures: the "time-swapping" method and the "equi-zenith" method, which give equivalent results.

The $\gamma$-ray emission from the Crab Nebula was detected with a significance of about 14.5 standard deviations in 800 days. Fig. 5 shows the three experimental points measured by ARGO-YBJ on the Crab Nebula energy spectrum, in perfect agreement with the known experimental results obtained by the HESS [7], MAGIC [8], and Tibet AS- $\gamma$ [9] experiments. 
Two remarkable flares from the MRK 421 active galactic nucleus were observed in the X-ray range by the ASM X-ray telescope on June 4-6 and June 11-13, 2008 [10]. ARGO-YBJ investigated

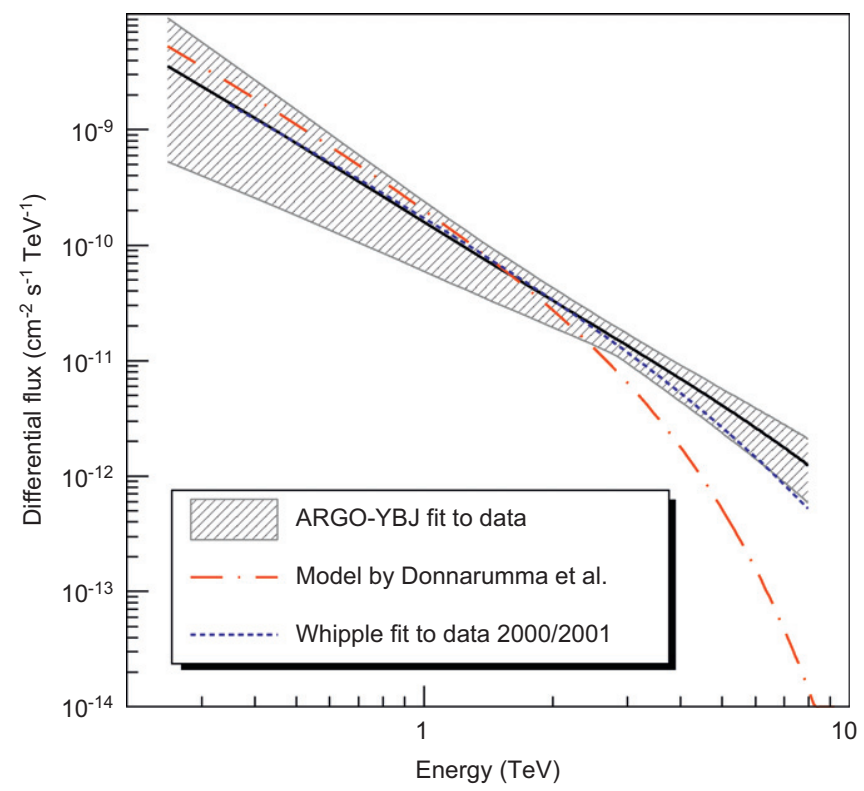

Fig. 7. Gamma-ray flux from MRK 421 measured by ARGO-YBJ on 2008, June 11-13 (solid line). The shaded band represents one standard deviation error. The dotdashed line shows the flux according to the model by Donnarumma et al. [10] for the second flare (June 12-13). The dotted line shows the spectrum measured by Whipple [13] during a previous flare of similar intensity.

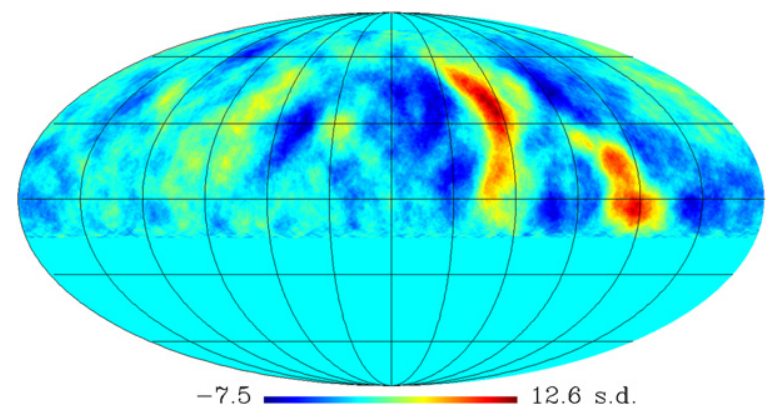

Fig. 8. Medium-scale sky map obtained by ARGO-YBJ. Two major excess regions are clearly visible.

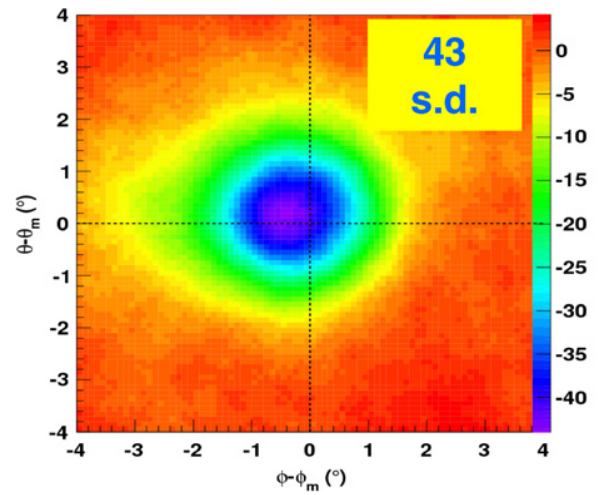

possible gamma emission on those days from the same source [11]. In this analysis, the requirement for the selected events was a number of hit pads greater than 100. Fig. 6 shows the significance map of the sky region around MRK 421 for the flare of June 11-13, 2008. The peak significance for this flare was 4.2 standard deviations.

Fig. 7 shows the ARGO-YBJ experimental results for the MRK 421 spectrum from the flare of June 11-13, 2008.

The ARGO-YBJ data fully satisfy the relation between the spectral index and the flux resulting from the Whipple measurements of a similar flare of MRK 421 [13], suggesting that this relation is an intrinsic property of the source.

A search for new possible $\gamma$-ray sources is in progress.

\section{ARGO-YBJ results in cosmic-ray physics}

The studies in cosmic-ray physics with ARGO-YBJ were focused so far on the following items: search for large-scale anisotropy at $\mathrm{TeV}$ energies, study of the moon shadow and the corresponding limit on the $\bar{p} / p$ flux ratio, proton-air interaction cross-section.

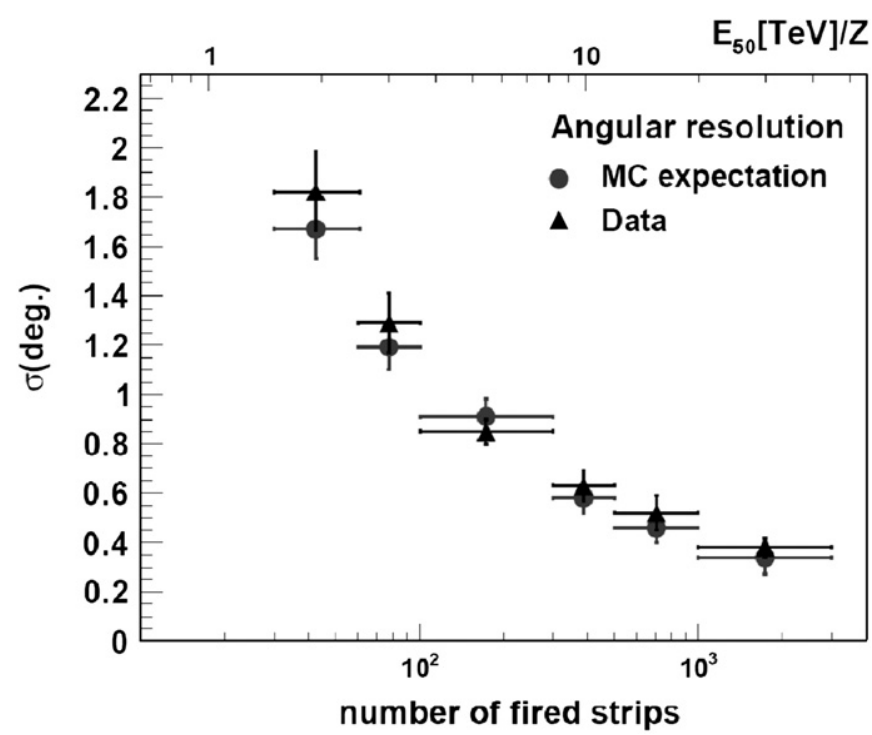

Fig. 10. Angular resolution of the ARGO-YBJ detector versus the hit number and the median energy scale.

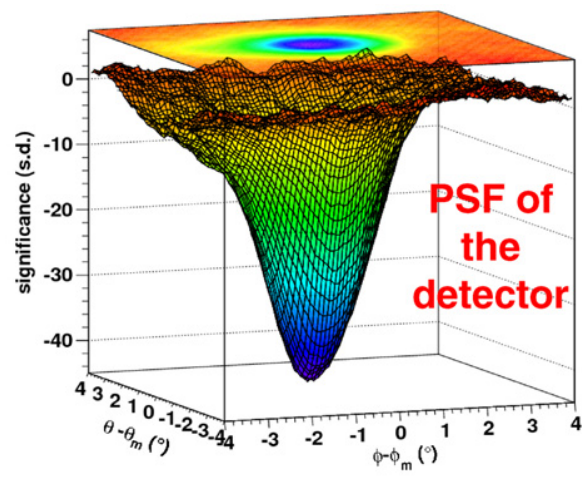

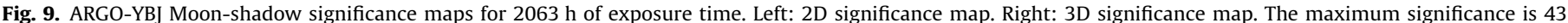
standard deviations. 


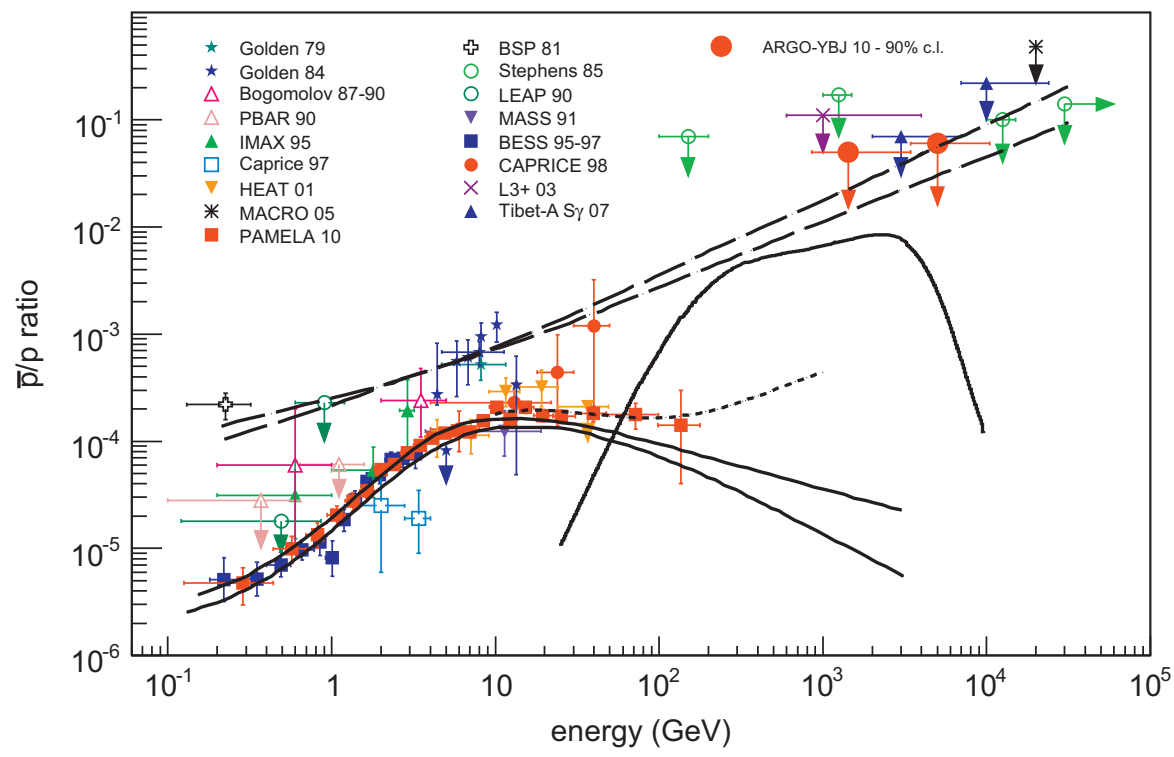

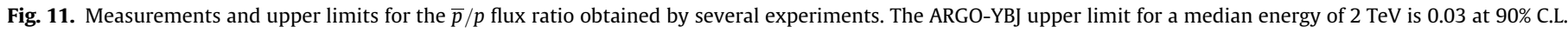

The first ARGO-YBJ significance map of the whole visible sky with cosmic rays was obtained using the data collected between day 311 in 2007 and day 220 in 2009. A number of hit pads greater than 40 were requested for this analysis (corresponding to a median energy of about $2 \mathrm{TeV}$ ) and the map, shown in Fig. 8 , was obtained by using a smoothing window radius of $5^{\circ}$.

Two major excess regions in the cosmic-ray flux appear. In principle this result is unexpected, since the interstellar magnetic field should randomize the arrival directions of $\mathrm{TeV}$ cosmic rays. However, possible explanations for flux anisotropy at $\mathrm{TeV}$ energies have been proposed by some authors [12].

An important subject of study in cosmic-ray physics is the shadowing effect exerted by the Moon on primary cosmic rays. Ground-based experiments can measure three crucial quantities related to this effect: the size, the position and the westward displacement of the deficit. From these measurements, information on the angular resolution, the pointing accuracy and the energy calibration of the detector respectively can be extracted. Concerning the last of these three issues, the basic procedure is based on the angular bending $\Delta \theta$ of a primary cosmic ray with energy $E$ and atomic number $Z$ in the geomagnetic field between the Moon and the Earth: $\Delta \theta \simeq 1.57^{\circ} \times Z / E(\mathrm{TeV})$.

The ARGO-YBJ analysis of the Moon shadow used data collected in the years 2006, 2007 and 2008, with 2063 h of exposure overall. Events with a number of hit pads greater than 60 and shower axis within $50^{\circ}$ from the vertical direction were used in this study.

A limit on the $\bar{p} / p$ flux ratio can be obtained by looking for a contribution of antiprotons in the Moon shadow. Fig. 9 shows the ARGO-YBJ significance map of the Moon-shadow deficit obtained with 2063 h of exposure time, with a maximum significance of 43 standard deviations with respect to the background.

The pointing angular resolution is related to the rms of the deficit: rms $\simeq \sigma \sqrt{1+(R / 2 \sigma)^{2}}$, where $R$ is the Moon angular radius.

Fig. 10 shows the angular resolution of the detector versus the number of hits and the scale for the median energy $E_{50}$. The overall angular resolution is less than $0.5^{\circ}$ for $E_{50} / Z$ greater than about $10 \mathrm{TeV}$.

Fig. 11 shows a comparison of the measurements of the $\bar{p} / p$ flux ratio obtained by satellite experiments at energies below $100 \mathrm{GeV}$, and the upper limits on this ratio obtained by ground-based experiments up to few tens of TeV. The present ARGO-YBJ upper limit is 0.03 at $90 \%$ C.L.

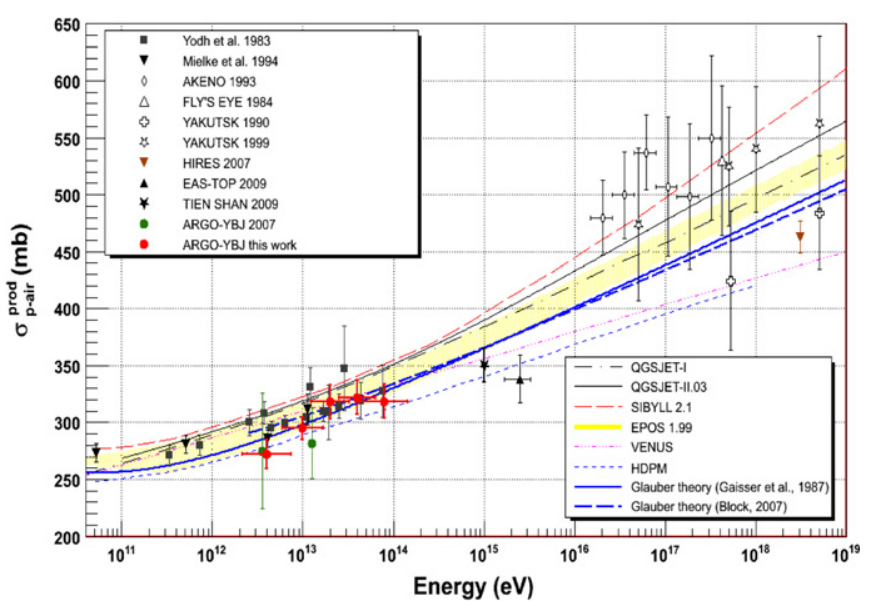

Fig. 12. Experimental results and theoretical predictions for the proton-air crosssection in the energy range between less than $10^{11}$ and $10^{19} \mathrm{eV}$. The ARGO-YB results, between $4 \times 10^{12}$ and $10^{14} \mathrm{eV}$, are in good agreement with the Glauber model prediction.

Another significant issue is the measurement of the proton-air interaction cross-section at energies ranging from a few $\mathrm{TeV}$ to $100 \mathrm{TeV}$. The expected flux of atmospheric showers versus the zenith angle $\theta$ is

$I(\theta)=I(0) \cdot e^{-\left(h_{0} / \Lambda\right)(\sec \theta-1)}$

where $h_{0}$ is the atmospheric depth of the experimental setup (606.7 g/ $\mathrm{cm}^{2}$ for ARGO-YBJ) and $\Lambda$ is the proton absorption length in air. It differs from the proton interaction length $\lambda_{\text {int }}$ mainly because of collision inelasticity, shower fluctuations and detector resolution. It can be shown that $\Lambda=k \lambda_{\text {int }}$, where $k$ must be determined by simulations. The proton-air interaction cross-section is given by

$\sigma_{p-A i r}=2.4 \times 10^{4} / \lambda_{i n t}\left(\mathrm{~g} / \mathrm{cm}^{2}\right)$.

This analysis was performed by selecting deep showers (smaller distance between the shower maximum and the detector) and exploiting the detector features (detailed space-time pattern) and location (high altitude). 
Fig. 12 shows the measurements of the proton-air cross-section performed by several experiments. The ARGO-YBJ results at five different energy values are in pretty good agreement with the prediction coming from the Glauber model [14].

\section{Conclusions}

The results obtained by ARGO-YBJ in the first two years of operation confirm the expectations, and the fact that the detector is running smoothly. Over $2 \times 10^{11}$ events have been collected so far. The additional analog readout of the ARGO-YBJ RPCs will extend the dynamical range of the experiment beyond $100 \mathrm{TeV}$, up to $1 \mathrm{PeV}$ and more. In addition, studies are in progress in view of the possibility of obtaining a better hadron-gamma discrimination by exploiting the unprecedented space-time structure of the air showers that the ARGO-YBJ detector can provide.

\section{References}

[1] R. Santonico, R. Cardarelli, Nucl. Instr. and Meth. A 377 (1981) 187; R. Cardarelli, R. Santonico, Nucl. Instr. and Meth. A 263 (1988) 200.

[2] C. Bacci, et al., Astropart. Phys. 17 (2002);

G. Aielli, et al., Astropart. Phys. 30 (2008) 85 .

[3] G. Aielli, et al., the ARGO-YBJ Collaboration, Nucl. Instr. and Meth. A 562 (2006) 92.

[4] G. Aielli, et al., Nucl. Instr. and Meth. A 409 (1998) 291.

[5] P. Camarri, et al., The detector control system for the ARGO-YBJ experiment, in: Proceedings of the 28th International Cosmic Ray Conference, Tsukuba, Japan, July 31 - August 7, 2003

[6] P. Camarri, on behalf of the ARGO-YBJ Collaboration, Nucl. Instr. and Meth. A 602 (2009) 668.

[7] F. Aharonian, et al., the HESS Collaboration, Astron. Astrophys. 457 (2006) 899.

[8] J. Albert, et al., the MAGIC Collaboration, Astrophys. J. 674 (2008) 1037.

[9] M. Amenomori, et al., the Tibet AS- $\gamma$ Collaboration, Astrophys. J. 692 (2009) 61.

[10] I. Donnarumma, et al., Astrophys. J. 691 (2009) L13.

[11] G. Aielli, et al., Astrophys. J. Lett. 714 (2010) L208.

[12] M. Salvati, B. Sacco, Astron. Astrophys. 4852 (2008) 527.

[13] F. Krennrich, et al., the WHIPPLE Collaboration, Astrophys. J. 575 (2002) L9.

[14] G. Aielli, et al., the ARGO-YBJ Collaboration, Phys. Rev. D 80 (2009) 092004. 